\section{Use of nuclear magnetic resonance (NMR) and metabonomic analysis of saliva in equine studies}

\author{
Sarah Ralston', István Pelczer², Elissa Lappostato' \\ and Teresa Soroka² \\ Department of Animal Science, Cook College, Rutgers University, \\ New Brunswick ${ }^{1}$ and Department of Chemistry, Princeton University, \\ Princeton, $\mathrm{NJ}^{2}$, USA
}

\section{Introduction}

High resolution Nuclear Magnetic Resonance Spectroscopy (NMR) is an exciting new tool in medicine. It can be used to detect and quantify low-molecular weight metabolites, drugs, and toxic agents in body fluids and tissues (Nicholsen et al. 2002, Nicholsen and Wilson 2003). It has been used in the pharmaceutical companies to screen for the by-products of new drugs and associated metabolic changes (Pelczer 2003, Pelczer 2005). In NMR spectroscopy magnetically active nuclei, such as 1H, 13C, 19F, 31P, 15N, etc., undergo a transition between energy states that are characteristic of the chemical structure of the molecules in which they are incorporated, creating a unique pattern for each component in the sample. Analysis of the chemical shifts and splitting patterns (J couplings) of samples' NMR spectra allow the determination of the molecular composition and relative concentrations of the components of the sample (Nicholsen et al. 2002, Nicholsen and Wilson 2003, Pelczer 2003, Pelczer 2005). Principal component analysis (PCA) of the spectra allows determination of clusters of like samples based on the complex array of organic molecules present, which is the basis for the new discipline of metabonomics (Nicholsen 2002).

All biofluids, such as urine, blood, saliva, semen, sweat, cerebrospinal fluid, bile, and others can be used in NMR studies (Nicholsen et al. 2002, Nicholsen and Wilson 2003, Pelczer 2003, Pelczer 2005). Saliva has been the subject of preliminary NMR analysis (Silwood et al. 2002) and used in studies of stress in horses (McGreevy and Pell 1999), however it is not known how reliable it would be for specific diagnostics and metabonomics in general. It is an abundant fluid that is more easily collected than blood or urine by simply inserting gauze pads into the horse's mouth and letting them chew on them. If metabonomic analysis of saliva could be shown to reliably detect characteristic metabolic differences related to stress or other conditions in horses it could be a very useful tool for future research and diagnostics that might even be extended to human studies.

We hypothesized that NMR analysis of saliva collected from weanling horses would detect differences between genders and stress levels, etc. Our objective was to determine if metabonomic and statistical analyses of NMR spectra of saliva samples would prove useful in detection of differences between a variety of groupings/circumstances in young horses.

\section{Materials and methods}

Saliva samples were collected from 12 draft cross horses (8 females, 4 males) between 4 to 11 months of age. Samples were taken at $07.30 \mathrm{~h}$ and 16.00 for the first 7 days after arrival at the facility (looking for indicators of stress), then weekly for the entire period. The horses were divided into two treatment groups and fed either a standard growth hay/concentrate or total mixed ration forage cubes in a simple cross design with 4 weeks for each trial and 4 weeks between trials. Before the treatments were initiated, however, the horses subjected to dominance testing in which each horse was paired with one of the other horses and placed in a neutral stall with a single bucket of feed. The horse that gained the greatest access to the feed was deemed the dominant animal of the pairing. Testing lasted 12 days, and every horse had been tested with every other horse in the herd. Saliva samples were collected immediately before and after each test. The colts were castrated between the feed trials and had samples taken before and after castration. The NMR metabolic profiles of both whole and filtered saliva samples were compared using PCA scores and loading plots (MATlab 7) of both the whole spectra and specific spectral regions (aliphatic 0.0-2.80 ppm, sugars 2.8-4.6 ppm, aromatic 6.00-10.5 $\mathrm{ppm}$ ) with respect to: sex, time after arrival, dominance ranking, pre- versus post-castration, metabolic reaction to deworming, relative reactivity of the horses to novel stimuli such as halter breaking and venipuncture. Principal component data were also subjected to Pearson's Correlation analysis to determine significant clustering and students T-tests where appropriate (Statistixs 8, Ocala, FL). Significance was set at $\mathrm{P}<0.05$.

\section{Results}

We have found clustering $(\mathrm{P}<0.05)$ due to metabolites in various regions of the spectra corresponding especially to amino-acids in the aliphatic region and aromatic compounds such as alkaloids and steroids, with respect to sex, pre- versus postcastration, morning versus afternoon sampling, and adaptation to the new environment (first 7 days). Specific metabolites causing the clusters have yet to be fully identified, though lactate and acetate were two components identified that were associated $(\mathrm{P}<0.05)$ with the clusterings due to stress, both post arrival and pre- and post-castration (Figure 1).

Similarly, one of the colts' (Apollo), full saliva spectra showed clustering $(\mathrm{P}<0.05)$ of the first 3 days after arrival (9/20/05-9/24/05) versus later dates due to peaks in the lactate and acetate regions in addition to metabolites at 1.24-1.28 ppm, 0.84-0.88 ppm, 3.24-3.28 ppm, 3.80$3.84 \mathrm{ppm}, 3.68-3.72 \mathrm{ppm}$ amd $4.08-4.12 \mathrm{ppm}$ (graphs not shown). 


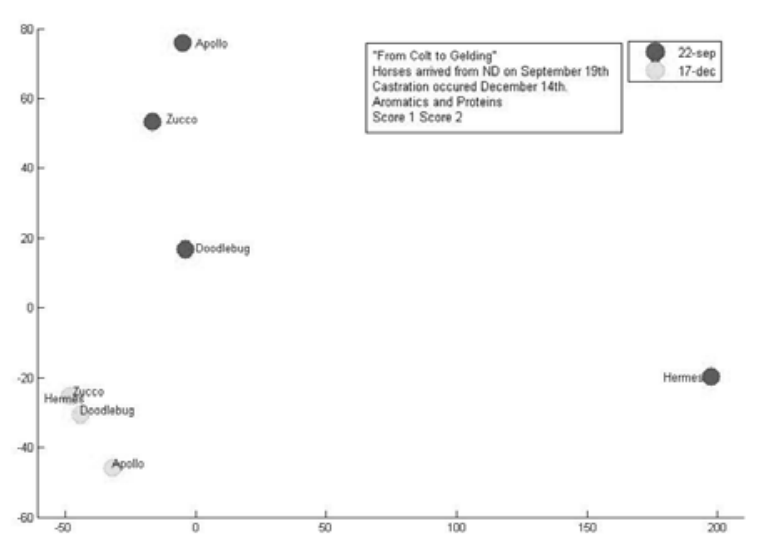

a

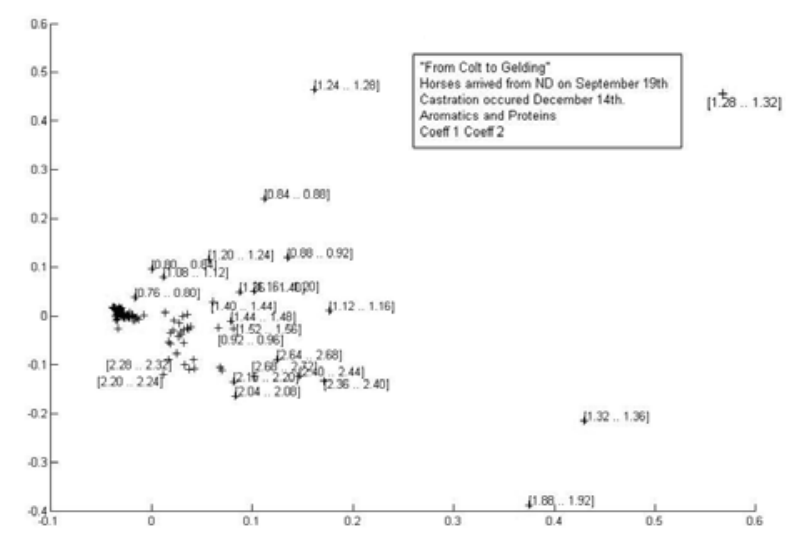

b

Fig 1 The "molecular scream"; $\mathbf{a}$ : the plot of PC1/PC2 scores of the complete saliva spectrum. The new geldings are clustered together the day after castration in the (lower left corner of the graph) in contrast to their scattered precastration positions. b: The loading plot for the same PC combination highlights that peaks in lactate $(1.3 \mathrm{ppm})$ and acetate $(1.9 \mathrm{ppm})$ are outliers (significant contributors to the clustering on the PCA plot), with peaks in three other outlier regions (1.24-1.28ppm, 0.84-0.88 ppm, 1.32-1.36 ppm) left to be identified.

\section{Conclusions}

The use of metabonomic and statistical analyses of NMR spectroscopy spectra of horse saliva may be useful in detection of complex metabolic differences both in acute instances and over time in controlled studies. This will permit identification of potential metabolic markers for diagnostic purposes and better understanding of the underlying biochemistry of metabolic events.

\section{References}

McGreevy P. D. and Pell S. M. (1999): A study of cortisol and betaendorphin levels in stereotypic and normal Thoroughbreds. Applied Animal Behavior Science 64, 81-90

Nicholson J. K., Connelly J., Lindon J. C. and Holmes E. (2002): Metabonomics: a platform for studying drug toxicity and gene function. Nature Review of Drug Discovery, 1, 153-161

Nicholson J. K. and Wilson I. D. (2003): Understanding 'Global' systems biology: metabonomics and the continuum of metabolism. Nature Review of Drug Discovery 2, 668-685

Pelczer I. (2003): Structural biology, ligand binding, metabomics the changing face of high-field, high-resolution NMR spectroscopy. Journal of. Molecular Structure (Theochem) 667, 499-505

Pelczer I. (2005): High-resolution NMR for metabomics. Current Opinion of Drug Discovery Development 8, 127-133

Silwood C. J. L., Lynch E., Claxson A. W. D. and Grootweld M.C. (2002): $1 \mathrm{H}$ and 13C NMR spectroscopic analysis of human saliva. Journal of Dental Research 81, 422-427 